\title{
Unmasking of severe hypertriglyceridaemia associated with antipsychotic therapy by atypical eruptive xanthomas
}

\author{
Magdalena Kiedrowicz' ${ }^{1}$ Radosław M. Kiedrowicz², Joanna Pietrzak-Halińska ${ }^{1}$, Mariusz Pietrzak ${ }^{3}$, Romuald Maleszka ${ }^{1}$ \\ 'Department of Dermatology and Venereology, Pomeranian Medical University Hospital No. 1, Police, Poland \\ ${ }^{2}$ Cardiology Department, Pomeranian Medical University Hospital No. 2, Szczecin, Poland \\ ${ }^{3}$ Department of Internal Medicine and Diabetology, Provincial Group Hospital, Szczecin, Poland
}

Adv Dermatol Allergol 2017; XXXIV (5): 507-509

DOI: https://doi.org/10.5114/ada.2017.71123

A 43-year-old male with a 2-year history of schizotypal and delusional disorders and known obesity with a body mass index (BMI) of $35 \mathrm{~kg} / \mathrm{m}^{2}$, presented with non-pruritic, painless skin lesions. Over the course of 18 months, following commencement of an antipsychotic therapy with quetiapine and risperidone, there was a progression from single papules on the patient's arms and legs to sudden eruption of multiple lesions scattered over the back and both sides of the arms and legs (Figure 1 A). These had been present for a number of weeks. Detailed dermatological examination revealed large, pink and polymorphous tuberous papules with a yellowish centre consistent with eruptive xanthomas (Figure $2 \mathrm{~A}$ ). A histopathology test of the skin lesion (Figures 2 B, C) showed extensive accumulation of lipid laden histiocytes confirming the diagnosis. The patient was otherwise asymptomatic. Serum biochemistry demonstrated hypercholesterolaemia (total cholesterol (TC) of $11.25 \mathrm{mmol} / \mathrm{l}$ ), severe hypertriglyceridaemia (fasting triglycerides (TG) of $35.3 \mathrm{mmol} / \mathrm{l}$ ) and long-standing hyperglycaemia (fasting glucose of $19.7 \mathrm{mmol} / \mathrm{l}, \mathrm{HbA}_{1 \mathrm{c}}$ of $10.9 \%$ ) that led to the diagnosis of a new onset of diabetes mellitus. Of note, his plasma LDL-cholesterol $(0.78 \mathrm{mmol} / \mathrm{l})$ and $\mathrm{HDL}$-cholesterol $(0.26 \mathrm{mmol} / \mathrm{l})$ levels were extremely low. Other laboratory tests including hepatic, renal, pancreatic and thyroid functions were normal. Abdominal ultrasound revealed significant liver steatosis. An algorithm [1, 2] based on plasma TG, TC and apolipoprotein (Apo) B $(71 \mathrm{~g} / \mathrm{l})$ excluded familial dysbetalipoproteinaemia (FD) with TC/Apo B ratio <6.2, TG/ApoB ratio > 10.0 and $A p o B / T C$ ratio $>0.15$. The patient's family history was positive for lipid disorders associated with type 2 diabetes in his father. A therapy with insulin, metformin and fenofibrate drugs was initiated and the patient was counselled on lifestyle changes including weight loss and a low-fat diet. A consulting psychiatrist decided not to discontinue the antipsychotic treatment. At 3 months' follow-up, the lesions had resolved with reddish discoloration at the primary sites (Figure 1 B). A significant improvement in lipid and glucose metabolism was observed (TC of $4.6 \mathrm{mmol} / \mathrm{l}$, TG of $10.1 \mathrm{mmol} / \mathrm{l}$, glucose of $6.1 \mathrm{mmol} / \mathrm{l})$, however in order to achieve the treatment goals [2], the therapy with quetiapine and risperidone was finally discontinued.

Xanthomas evolve as clusters of foam cells in the connective tissue of the skin, tendons and fasciae. Foam cells are formed from macrophages as a consequence of gradual intracellular accumulation of lipids. The clinical picture of xanthomas is variable. These are often associated with dyslipidaemias and can be pathognomonic in some cases. Eruptive xanthomas can call attention to severe hypertriglyceridaemia, especially in patients with newly diagnosed or decompensated diabetes mellitus. Tuberous xanthomas usually occur in patients with inherited dyslipidaemias such as FD and rarely in cases of secondary dyslipoproteinaemias. These mostly manifest themselves in the skin over joints (elbows, knees, hand joints and feet) and on the extensor surfaces of extremities or on the buttocks and in skin creases. Tuberous xanthomas are prominent yellowish papules, from $3 \mathrm{~mm}$ to several centimetres in size and often pruritic [3].

Severe hypertriglyceridaemia presented in adulthood with a coincidental cause of dyslipidaemia associated with obesity, diabetes mellitus and drugs affecting the metabolism of TG-rich lipoproteins along with tuberous xanthomas was highly suggestive of FD. It is an inherited mixed hyperlipidaemia caused by an abnormality in Apo $E[1,2,4]$. Despite the presented clinical picture it was reliably excluded by laboratory plasma tests. In this case, dyslipidaemia manifested itself exclusively by

Address for correspondence: Magdalena Kiedrowicz MD, PhD, Department of Dermatology and Venereology, Pomeranian Medical University Hospital No. 1, 2 Siedlecka St, 72-010 Police, Poland, phone: +48 510034 332, e-mail: magkied@poczta.onet.pl Received: 13.03.2016, accepted: 27.06.2016. 

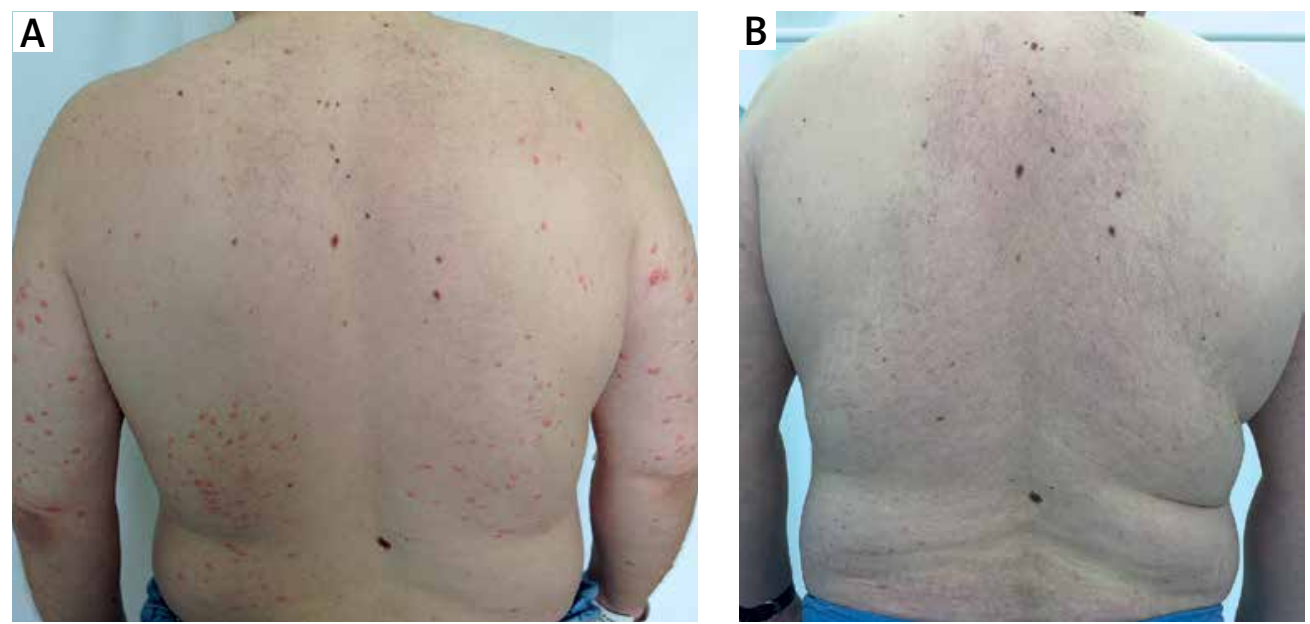

Figure 1. A - Clinical presentation of eruptive xanthomas. B - Regression of the skin lesions after 3 month hypolipidemic treatment
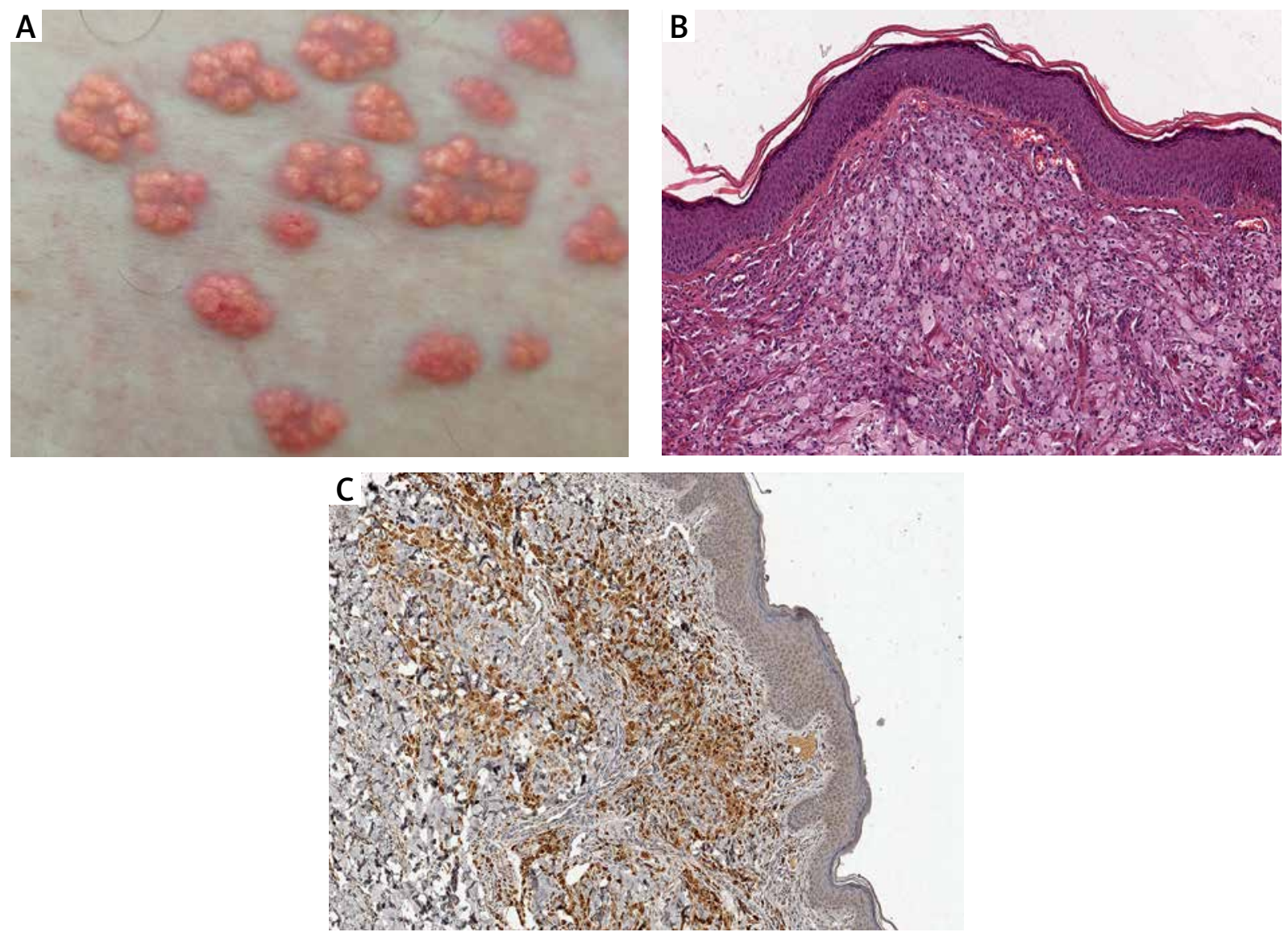

Figure 2. Eruptive xanthomas - large, pink and polymorphous papules with a yellowish centre (A). Histopathological examination of eruptive xanthomas showing extensive accumulation of lipid laden histiocytes. H-E, Magnification 200× (B, C)

atypical eruptive tuberous xanthomas, which had not appeared in the common body locations. The mechanism of this phenomenon remains unclear as a sudden eruption of skin lesions.
We strongly believe that severe hypertriglyceridaemia and hyperglycaemia were caused by the use of antipsychotic agents or alternatively due to a combination of the therapy and undiagnosed type 2 diabetes. Quetiap- 
ine has known metabolic side effects such as weight gain, significant increase in TC, TG and glucose plasma levels that can occur in more than $50 \%$ of patients [5]. Moreover, quetiapine is known to be associated with a more rapid onset of metabolic disturbances than other antipsychotics. Previously observed time periods to the onset of hyperglycaemia and hypercholesterolemia among patients treated with the drug was 17.5 and 15.8 months, respectively [6]. Common risperidone metabolic side effects include increased weight and hyperglycaemia [5]. However, we cannot exclude that the antipsychotic therapy exacerbated underlying lipoprotein metabolic abnormality secondary to undiagnosed diabetes.

This paper indicates that dermatologists can play a key role in the diagnostic process of unmasking dyslipidaemias. The ability to recognize clinical forms of xanthomas is an important step in a detailed examination, however one should remember that the clinical picture of these skin lesions can be variable and therefore not always indicative of a particular type of metabolic disturbances. Antipsychotic-induced metabolic adverse effects, such as hyperglycaemia and dyslipidaemia should be of particular concern, especially these side effects may evolve to a high-risk state for future cardiovascular morbidity and mortality.

\section{Conflict of interest}

The authors declare no conflict of interest.

\section{References}

1. Sniderman A, Trembley A, Bergeron J, et al. Diagnosis of type III hyperlipoproteinemia from plasma total cholesterol, triglyceride and apolipoprotein B. J Clin Lipidol 2007; 1: 256-63.

2. Reiner Z, Catapano AL, De Backer G, et al. ESC/EAS Guidelines for the management of dyslipidaemias: the Task Force for the management of dyslipidaemias of the European Society of Cardiology and the European Atherosclerosis Society. Eur Heart I 2011; 32: 1769-818.

3. Zak A, Zeman M, Slaby A, Vecka M. Xanthomas: clinical and pathophysiological relations. Biomed Pap Med Fac Univ Palacky Olomouc Czech Repub 2014; 158: 181-8.

4. Blom DJ, Byrnes P, Jones S, Marais AD. Dysbetalipoproteinaemia - clinical and pathophysiological features. S Afr Med J 2002; 92: 892-7.

5. Rummel-Kluge C, Komossa K, Schwarz S, et al. Head-tohead comparisons of metabolic side effects of second generation antipsychotics in the treatment of schizophrenia: a systematic review and meta-analysis. Schizophr Res 2010; 123: 225-33.

6. Letourneau G. Quetiapine linked to more rapid onset of metabolic disturbances in elderly patients. Am J Geriatr Psychiatry 2010; 18 Suppl. 3, abstr. 20; AAGP Annual Meeting 2010, USA. 